\title{
Semi-supervised Gaussian Process for Automated Enzyme Search
}

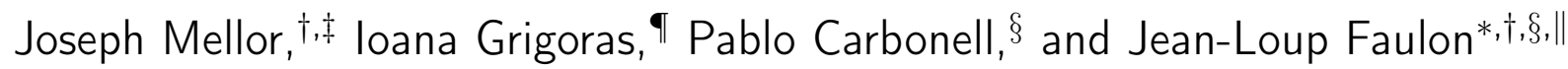

$\lceil$ School of Chemistry, University of Manchester, Manchester, UK

$\ddagger$ Manchester Institute of Biotechnology, University of Manchester, Manchester, UK

【iSSB, institute of Systems and Synthetic Biology, CNRS, University of

Évry-Val-d'Essonne, Évry, FR

$\S S Y N B I O C H E M$ Centre, Manchester Institute of Biotechnology, University of Manchester, Manchester, UK

||MICALIS Institute, INRA, Jouy en Jossas, FR

E-mail: jean-loup.faulon@manchester.ac.uk

\section{Supplementary Material}



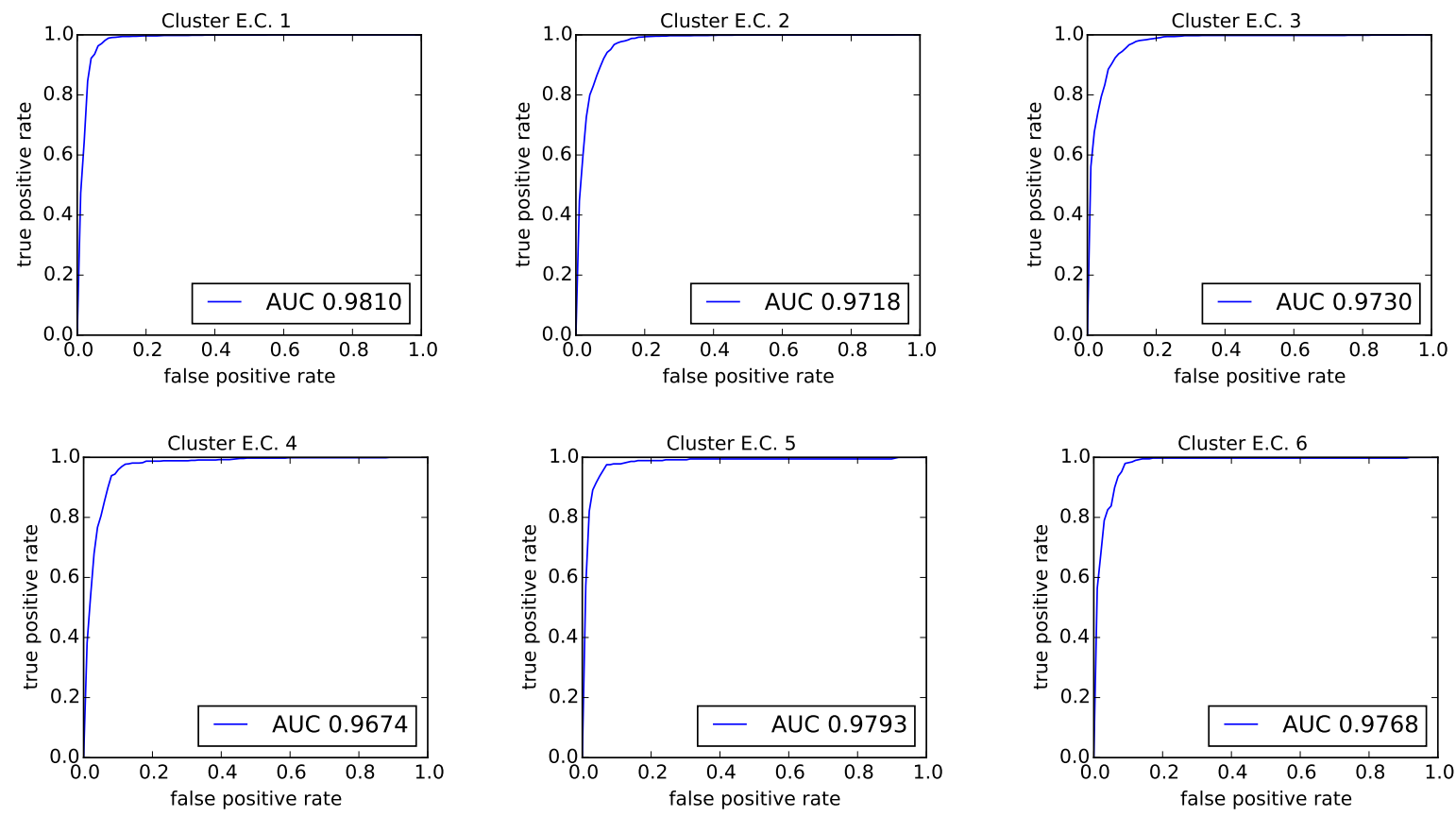

Figure S1: Mean Receiver Operating Characteristic curves for the 6 individual clusters formed using the top level E.C. number. The results are from a crossfold validation where unlabelled data, generated as described in the methods section, is assumed to be negative in the test fold. Each E.C. class contained equal numbers of positive and unlabelled data. Class 1 contained 3315 of each; class 2 contained 5404 of each; class 3 contained 4615 of each; class 4 contained 1614 of each; class 5 contained 956 of each and class 6 contained 906 of each. 

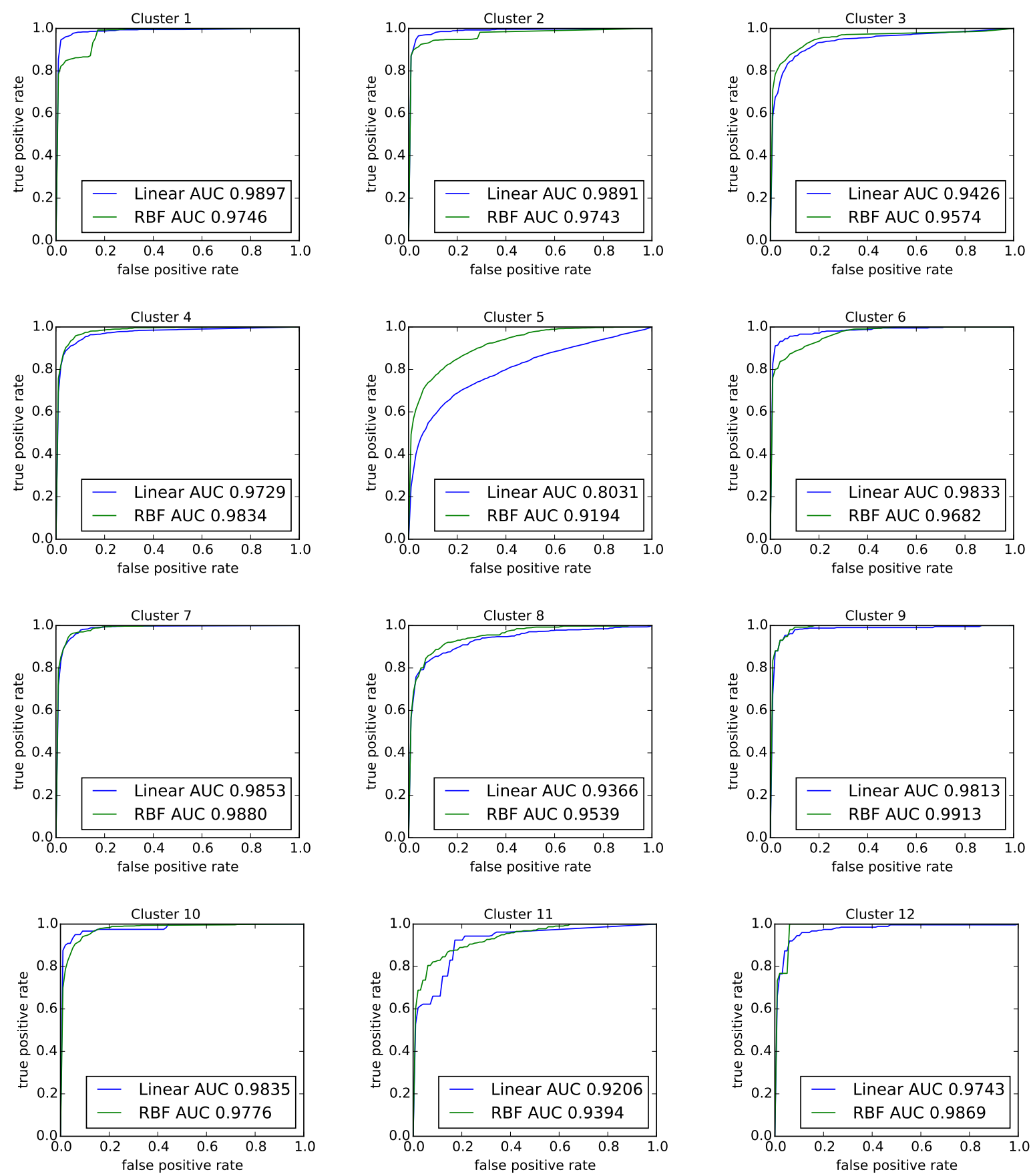

Figure S2: Mean Receiver Operating Characteristic curves for the 12 individual clusters formed using DBSCAN. The results are from a crossfold validation where unlabelled data, generated as described in the methods section, is assumed to be negative in the test fold. The number of positive/unlabelled data for each cluster was: 1581 for cluster 1, 1084 for cluster 2, 1749 for cluster 3, 2636 for cluster 4, 3949 for cluster 5, 519 for cluster 6, 1387 for cluster 7, 806 for cluster 8, 518 for cluster 9, 1206 for cluster 10, 527 for cluster 11, and 552 for cluster 12 . 